\title{
Humanities
}

\section{Grammar Lessons}

Stroke is a verb

Stroke is a noun

The word implies an action

or a thing

Discussed like it's tangible

but it's a deficit

A lack of blood

- insufficient funds to the brain

You can stroke

You can have a stroke

Acute ischemic stroke

Stroke out

He's stroking

Shit - he's stroking!

Aphasic

Bradycardic

Emesis

Limp limbs from hemiparesis

Unconscious

Fibrinolytic checklists

Inject the thrombolytic

Stroke is a verb.

Stroke is a noun.

Sarah Fraser

Class of 2014,

Faculty of Medicine,

Dalhousie University 